Ann. Zootech., I06r, $10(2)$, I4I - I42.

\title{
RELATIONS ENTRE L'IMPORTANCE DU SQUELETTE ET QUELQUES MESURES DE CONFORMATION CHEZ LE POULET DE CHAIR.
}

\author{
F. H. RICARD \\ Station de Recherches avicoles, \\ Domaine expérimental du Magneraud, Saint-Pierre d'Amilly (Charento-Maritime)
}

De nombreux chercheurs ont estimé l'importance relative des os et de la viande dans les carcasses de poulets. Mais aucun, à notre connaissance, n'a essayé d'évaluer le pourcentage d'os directement à partir de mesures de conformation. Le but de cette note est d'apporter quelques données sur ce sujet.

\section{MATÉRIEL ET MÉTHODES}

L'étude a porté sur $I_{5} 8$ coquelets de la même souche expérinentale. Les poulets provenaient de 8 lots éclos du 22 Juin au Io Août 1960 , élevés dans les 8 cases d'une même poussinière ; ils ont reçu la même alimentation et ont été sacrifiés au même âge ( 87 jours).

Nous avons travaillé sur les carcasses prêtes à cuire : carcasses effilées avec tête coupée an ras du crâne, pattes déjointées à l'articulation tibia-tarsométatarse, tous les organes internes enlevés sauf les reins et les poumons. Toutes les carcasses ont cuit à la vapeur pendant 75 minutes dans le panier d'un autocuiseur, en fin d'après-midi. Elles étaient laissées toute la nuit à la température du laboratoire. Le lendemain, les os étaient soigneusenent séparés de la viande, raclés et immédiateinent pesés au demi-gramme près. Ce poids a été ensuite rapporté au poids de la carcasse prête à cuire, ce qui a donné un rendement en os " $\mathrm{R}_{o s}$ ".

Par ailleurs les mesures suivantes avaient été prises : complet.

- Poids vif à 8 semaines et immédiatement avant labattage, après environ $15 \mathrm{~h}$. de jeûne

- Le poids de la carcasse effilée. effilée.

- L'angle de poitrine mesuré à l'aide d'un anglemètre AP-8, à 8 semaines et sur la carcasse

- La longueur du bréchet mesurée à 8 semaines et sur la carcasse à l'aide d'un mètre-ruban.

- La longueur du tarse (L) mesurée au mm près avec un pied à coulisse sur la patte recourbée, entre la partie externe de l'articulation tibia-tarse et la sole plantaire, à 8 semaines et sur la carcasse effilée.

-- Le petit diamètre du tarse (d) mesuré au pied à coulisse au dixième de mm près, à 8 semaines et sur la carcasse effilée.

Puis les rapports suivants ont été calculés :

- rapport $L / \mathrm{d}$ qui donne une idée de la finesse intrinsèque de la patte.

-- rapport Poids vif $\left./ \mathrm{L} \times \mathrm{d}^{2}{ }^{1}\right)$ qui donne une estimation du rapport poids total /poids squelette. rapport.

- rapport poids carcasse effilée/poids des 2 pattes qui donne une autre estimation du même

() Rapport proposé par L. P. COCHEZ, Directeur du Domaine du Magneraud. 


\section{RÉSULTATS}

$I^{0}$ Caractéristiques du rendement en os " $\mathbf{R}_{o s}$ ":

Moyenne ........ II,00 p. Ioo (valeurs extrêmes 8,6 et I4,0)

écart-type ...... $0,88 \mathrm{p}$. I00

$2^{\circ}$ Corrélations phénotypiques entre “ $\mathrm{R}_{o s}$ ” et les mesures de conformation:

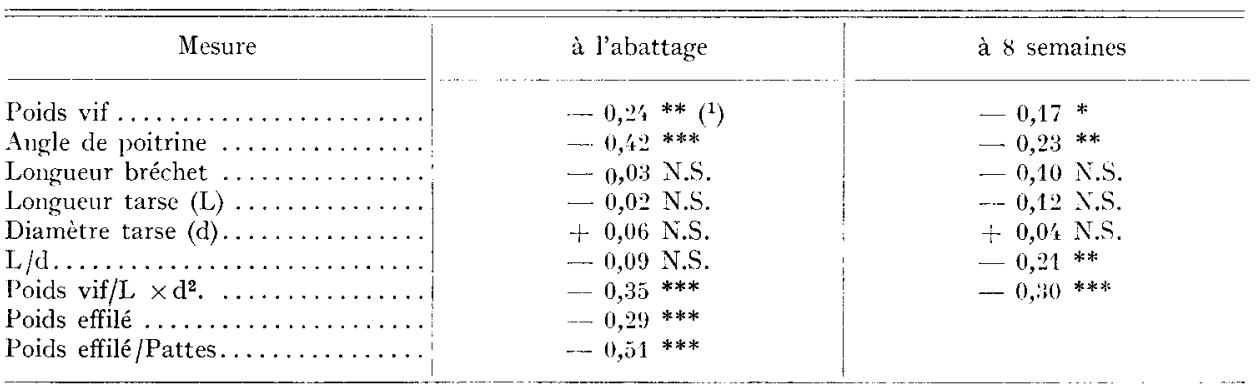

(1) N.S. : Valeur non significativement différente de zéro

* : Valeur différente de zéro au seuil 5 p. cent

** : Valeur différente de zéro au seuil i p. cent

*** : Valeur différente de zéro au seuil o, I p. cent

\section{DISCUSSION}

La variabilité du pourcentage d'os est relativement importante (coefficient de variation : 8 p. Ioo), ce qui suggère une amélioration possible du rapport viande os par la sélection.

Pour estimer ce pourcentage d'os, une seule mesure linéaire ne suffit pas, la corrélation avec la longueur du bréchet, la longueur du tarse ou l'épaisseur du tarse n'est pas significative.

Le rapport $\mathrm{L} / \mathrm{d}$ est en corrélation légèrement négative avec le pourcentage d'os mais seule la valeur à 8 semaines est significative. De même le poids vif n'est que faiblement représentatif de l'importance du squelette.

Par contre l'angle de poitrine mesuré sur la carcasse, le rapport poids vif/ $L \times \mathrm{d}^{2}$ et le rapport poids effilé/poids des pattes, permettent une bonne estimation du pourcentage d'os : plus la valeur de ces critères est élevée, plus la proportion d'os est faible.

Ceci suggère l'utilisation directe de ces caractères dans la sélection pour un poulet à squelette fin.

Rę̧u en avil ig6r.

\section{SUMMARY}

RELATIONSHIPS BETWEEN BONE YIELI) AND SOME CONFORMATION TRAITS IN BROILERS

An attempt vas made to appraise the bone yield of ready-to-cook male broiler carcasses. 158 twelve-weeks old chickens of the same strain were used. The mean was found to be I I percent and the standard deviation 0,88 percent. Phenotypic correlations were computed between bone yield and several conformation traits. Interesting negative values were found for carcass breast angle $(-0,42)$, dressed weight $(-0,29)$, live weight /shank length $\times$ (shank diameter) ${ }^{2}$ ratio $(-0,35)$ and dressed weight/shanks + feet weight ratio $(-0,5 \mathrm{I})$. 